Rev. Inst. Flor. v. 30 n. 1 p. $39-45$ jun. 2018

http://dx.doi.org/10.24278/2178-5031.201830103

ISSN impresso 0103-2674/on-line 2178-5031

\title{
DIFERENTES TIPOS DE SUBSTRATO PARA A PRODUÇÃO DE MUDAS DE AÇAÍ Euterpe oleracea MART. ${ }^{1}$
}

\section{DIFFERENT TYPES OF SUBSTRATES FOR THE PRODUCTION OF AÇAÍ SEEDLINGS Euterpe oleracea MART.}

\author{
Rhonan Martins de SOUSA ${ }^{2,5}$; Paulo Henrique Aquino MARINHO'; \\ Ana Beatriz Marques HONÓRIO ${ }^{3}$; Marcelo Ribeiro VIOLA ${ }^{4}$; Marcos Vinicius Giongo ALVES²; \\ Priscila Bezerra de SOUZA²
}

\begin{abstract}
RESUMO - Euterpe oleracea Mart., conhecida popularmente por açaí, influencia e movimenta grandes mercados na Região Amazônica, principalmente pela extração da polpa e do palmito. Portanto, objetivou-se avaliar a influência de diferentes tipos de substrato na produção, no desenvolvimento e na qualidade de mudas de Euterpe oleracea Mart. O experimento foi conduzido no viveiro da UFT, com delineamento em blocos ao acaso, com sete tratamentos e quatro repetições, sendo sete plantas por repetição. Os substratos utilizados foram: T1- substrato comercial Bioplant $^{\circledR}$ (100); T2- esterco bovino mais casca de arroz carbonizada (50:50); T3- esterco bovino mais areia (50:50); T4- esterco bovino mais areia (25:75); T5- substrato comercial mais casca de arroz carbonizada (25:75); T6- substrato comercial mais esterco bovino (25:75); e T7- areia mais esterco bovino (25:75). As avaliações foram feitas aos 60, 90 e 120 dias. Os parâmetros avaliados foram: altura $(\mathrm{H}, \mathrm{em} \mathrm{cm})$, diâmetro do coleto $(\mathrm{DC}, \mathrm{em} \mathrm{mm})$, relação altura/diâmetro (H/DC), massa seca da parte aérea (MSPA, em g planta-1), massa seca da raiz (MSR, em g planta ${ }^{-1}$ ), massa seca total (MST), relação entre a massa seca da parte aérea e a massa seca da raiz (RMSPAR, em g planta ${ }^{-1}$ ) e índice de qualidade de Dickson (IQD). Aos 120 dias, $\mathrm{T} 6$ apresentou melhores resultados nos parâmetros DC $(5,33 \mathrm{~mm})$ e H $(30,16 \mathrm{~mm})$. O T5 obteve melhores resultados para MSR $(4,51 \mathrm{~g})$ e IQD $(1,41)$. O parâmetro MSPA foi melhor no T1 (4,77 g); já o T7 conseguiu melhores resultados para MST (9,06 g). Portanto, os tratamentos com esterco bovino em sua composição, principalmente nas proporções de $50 \%$ de esterco bovino mais $50 \%$ de areia (T3), $25 \%$ de areia mais $75 \%$ de esterco bovino (T7) e $25 \%$ de substrato comercial Bioplant ${ }^{\circledR}$ mais $75 \%$ de esterco bovino (T6), proporcionaram os melhores crescimentos em altura e diâmetro do coleto, bem como aumentaram a produção de massa seca total em mudas de Euterpe oleracea Mart.
\end{abstract}

Palavras-chave: Arecaceae; crescimento inicial; qualidade de mudas.

\footnotetext{
${ }^{1}$ Recebido para análise em 07.12.2017. Aceito para publicação em 14.05.2018

${ }^{2}$ Universidade Federal do Tocantins, Campus de Gurupi, Rua Badejós, Lote 7, Chácaras 69/72, Zona Rural, Caixa Postal 66, CEP 77402-970, Gurupi, TO, Brasil.

${ }^{3}$ Universidade Estadual Paulista “Júlio de Mesquita Filho", Instituto de Biociências, Departamento de Botânica, Campus Rubião Junior, CEP 18618-689, Botucatu, SP, Brasil.

${ }^{4}$ Universidade Federal de Lavras, Departamento de Engenharia, Caixa Postal 3037, CEP 37200-000, Lavras, MG, Brasil.

${ }^{5}$ Autor para correspondência: Rhonan Martins de Sousa - rohsousa@hotmail.com
} 


\begin{abstract}
Euterpe oleracea Mart. known popularly by açaí, influences and moves great markets in the Amazon region, mainly by the extraction of the pulp and palmetto. Therefore, the aimed of this study was to evaluate the influence of different types of substrates on the production, development, and quality of Euterpe oleracea Mart. seedlings popularly known as açaí. The experiment was conducted in the nursery of UFT, with randomized block design, with 7 treatments and 4 replicates, being 7 plants per replicate. The substrates used were: T1-Bioplant ${ }^{\circledR}$ commercial substrate (100), T2-Bovine Manure + Carbonized rice husk (50:50), T3-Bovine Manure + sand (50:50), T4-Bovine Manure + sand: T5-Commercial substrate + Carbonized rice husk (25:75), T6-Commercial Substrate + Bovine Manure (25:75) and T7-Sand + Bovine Manure (25:75). The evaluations were done at 60,90 and 120 days. The parameters evaluated were: height $(\mathrm{H} ; \mathrm{cm})$, collection diameter $(\mathrm{DC} ; \mathrm{mm})$, height/diameter ratio $(\mathrm{H} / \mathrm{DC})$, dry mass of the aerial part (MSPA; g plant ${ }^{-1}$ ), root dry mass (MSR; g plant ${ }^{-1}$ ), total dry mass (MST), relation between the aerial part dry mass and root dry mass (RMSPAR; g plant-1) and Dickson quality index (IQD). At 120 days, T6 presented better results in the parameters DC $(6,62 \mathrm{~mm})$ and $\mathrm{H}(30,16 \mathrm{~mm})$. The T5 obtained better results for MSR $(4,51 \mathrm{~g})$ and IQD $(1,41)$. The MSPA parameter was better in T1 (4,77 g), and T7 had better results in MST (9,06 g). Therefore, the treatments with bovine manure in its composition, in the proportions of $50 \%$ of Bovine Manure $+50 \%$ of Sand (T3), $25 \%$ of Sand $+75 \%$ of Bovine Manure (T7) and $25 \%$ of Bioplant ${ }^{\circledR}$ Commercial Substrate $+75 \%$ of Bovine Manure (T6), provided the best growth in height and collection diameter, and increased dry mass production in Euterpe oleracea Mart seedlings.
\end{abstract}

Keywords: Arecaceae; initial growth; quality of seedlings.

\section{INTRODUÇÃO}

A distribuição, a abundância, a produtividade e a diversidade fazem com que a família Arecaceae se destaque entre as famílias de espécies florestais, sendo de grande importância alimentar, medicinal, sociocultural e econômica (Zambrana et al., 2007). Entre as várias espécies da família Arecaceae, a Euterpe oleracea Mart., conhecida popularmente como açaizeiro, é uma planta nativa da região Norte do Brasil, ocorrendo de forma espontânea nos Estados do Amazonas, Pará, Amapá, Maranhão, Tocantins e Mato Grosso (Conforto e Contin, 2009; Mendonça et al., 2014).

No Estado do Pará, o açaizeiro se destaca pela sua contribuição econômica para a movimentação de grandes mercados nessa Região Amazônica, principalmente por causa da comercialização de seu fruto, do qual, por meio da maceração, obtém-se uma bebida concentrada conhecida por açaí (Neves et al., 2015; Rodrigues et al., 2016). A presença de caules cespitosos permite extrair dessa planta um segundo produto comercial, o palmito, que é utilizado tanto na culinária brasileira como na internacional. Além disso, o seu uso alimentar é comum desde épocas remotas (Oliveira et al., 2017).
O Brasil se encontra como o maior produtor, consumidor e exportador do fruto da espécie Euterpe oleracea Mart. (açaí), além de ser o maior produtor e consumidor de palmito (Portinho et al., 2012). Nesse sentido, produzir mudas de qualidade e renovar os plantios do açaizeiro são de grande importância para economia do país. Segundo Melém Junior e Queiroz (2011), a valorização e a crescente demanda pela polpa do açaí causaram consequências notáveis na maneira como a espécie é manejada, sempre com o objetivo de aumentar a produção. Carvalho (2016) afirma que a alta demanda dos produtos do açaizeiro se tornou essencial para o aumento da produção de mudas e que a germinação rápida e uniforme e a emergência imediata de plântulas são algumas características essenciais para a produção delas.

Para que essas características sejam alcançadas na produção das mudas, é preciso escolher um substrato eficiente que tenha boa aeração, drenagem, retenção de água, disponibilização de nutrientes, fácil aquisição e custo acessível (Souza Júnior et al., 2008).

Com base no exposto, objetivou-se avaliar a influência de diferentes tipos de substrato na produção, no desenvolvimento e na qualidade de mudas de Euterpe oleracea Mart., popularmente conhecida por açaí. 


\section{MATERIAL E MÉTODOS}

O estudo foi conduzido no viveiro florestal localizado na Universidade Federal do Tocantins (UFT), campus de Gurupi, entre os meses de fevereiro e junho de 2017. O município de Gurupi está localizado na região sul do Estado do Tocantins, sob as coordenadas geográficas $11^{\circ} 44^{\prime} 08^{\prime \prime}$ de latitude Sul e $49^{\circ} 02^{\prime}$ '58' de longitude Oeste, a $280 \mathrm{~m}$ de altitude. Seu clima, de acordo Thornthwaite, é classificado como (C2wA "a"), ou seja, subúmido com moderada deficiência hídrica no inverno e precipitação variando entre 1.300 e 1.700 mm (SEPLAN, 2012; Sousa, 2016).

O experimento foi conduzido em delineamento de blocos ao acaso (DBC), com sete tratamentos e quatro repetições, sendo sete plantas por repetição. Os substratos utilizados foram: T1- substrato comercial Bioplant $^{\circledR}$ (100); T2- esterco bovino mais casca de arroz carbonizada (50:50); T3- esterco bovino mais areia (50:50); T4- esterco bovino mais areia (25:75); T5- substrato comercial mais casca de arroz carbonizada (25:75); T6- substrato comercial mais esterco bovino (25:75); e T7- areia mais esterco bovino (25:75).

Os frutos de Euterpe oleracea Mart. foram coletados e beneficiados no mês de janeiro de 2017, no município de Parauapebas, sudeste do Estado do Pará. Depois, foram semeadas três sementes em sacos pretos de polietileno com dimensões $22 \times 11 \mathrm{~cm}$ (comprimento x largura) sem nenhuma metodologia para superação de dormência. Em seguida, os sacos foram organizados em canteiros suspensos aproximadamente $1 \mathrm{~m}$ do solo, em uma casa de sombra coberta com sombrite com $50 \%$ da luminosidade. Após a semeadura, foram realizadas manualmente duas irrigações diárias. Logo após a emergência das plântulas de açaí, em torno do $52^{\circ}$ dia realizou-se um desbaste, deixando apenas uma plântula por recipiente, ou seja, a mais vigorosa.

Aos 60, 90 e 120 dias após a germinação, foram avaliados a altura da parte aérea $(\mathrm{H}, \mathrm{em} \mathrm{cm})$, com régua graduada ao nível do substrato até o ápice da parte aérea $(\mathrm{cm})$, e o diâmetro do coleto (DC, em mm), com um paquímetro digital com precisão de $0,01 \mathrm{~mm}$. A relação $\mathrm{H} / \mathrm{DC}$ foi determinada pela divisão entre os dois parâmetros: altura e diâmetro do coleto, individualmente de cada muda.
No final do experimento, foram obtidos os parâmetros peso da massa seca da raiz (MSR), peso da massa seca da parte aérea (MSPA) e peso da massa seca total (MST), com as raízes sendo separadas da parte aérea, lavadas em peneiras e, em seguida, acondicionadas em sacos de papel Kraft; na sequência, foram colocadas para secar em estufa com circulação forçada de ar a $72^{\circ} \mathrm{C}$ até a obtenção de peso constante.

Em seguida, as plântulas foram pesadas em uma balança analítica eletrônica, com precisão de $0,01 \mathrm{~g}$. A massa seca total (MST) foi obtida por meio da soma das duas medições (MST = MSPA + MSR, em $\left.\mathrm{g} \mathrm{planta}^{-1}\right)$. Além disso, foram determinados a relação entre a massa seca da parte aérea e a massa seca do sistema radicular $(\mathrm{RMSPAR}=\mathrm{MSPA} / \mathrm{MSR}$, em g planta $^{-1}$ ) e o índice de qualidade de Dickson (IQD), definido pela expressão: IQD $=$ MST / [(H / DC $)+$ (MSPA / MSR)].

As análises estatísticas foram realizadas pelo software Sisvar (Ferreira, 2011), em que os dados foram submetidos à análise de variância, e as médias dos tratamentos utilizados, comparadas pelo teste Tukey a $5 \%$ de probabilidade.

\section{RESULTADOS E DISCUSSÃO}

O diâmetro do coleto (DC) apresentou variações quanto ao desenvolvimento das mudas de Euterpe oleracea aos 60, 90 e 120 dias. Verificou-se que, aos 60 dias após o plantio, o T4, composto por esterco bovino mais areia (25:75), foi o que apresentou melhor desenvolvimento, com 2,93 mm. Entretanto, não houve diferenças estatísticas para T1, T3, T6 e T7, mantendo médias iguais conforme o teste aplicado (Tabela 1). O T2 e o T5 foram considerados os piores, com 2,62 e 2,49 mm, respectivamente. De acordo com Medeiros et al. (2007), substratos constituídos por areia lavada mais alguma fonte de matéria orgânica proporcionam maior desenvolvimento e comprimento da raiz, o que contribui diretamente para o desenvolvimento da planta, pois possibilita melhor absorção e fixação da muda no substrato, principalmente nos estágios iniciais de desenvolvimento. 
SOUSA, R.M. et al. Produção de mudas de açaí..

Tabela 1. Valores obtidos aos 60, 90 e 120 dias após a semeadura de Euterpe oleracea Mart. para altura da planta (H), diâmetro de coleto (DC) e relação altura/diâmetro (H/DC).

Table 1. Values obtained at 60, 90 and 120 days after sowing of Euterpe oleracea Mart. for: plant height (H), collection diameter (DC) and height-to-height ratio (H/DC).

\begin{tabular}{|c|c|c|c|c|c|c|c|c|c|}
\hline \multirow{2}{*}{ Tratamento } & \multicolumn{3}{|c|}{60 dias } & \multicolumn{3}{|c|}{90 dias } & \multicolumn{3}{|c|}{120 dias } \\
\hline & $\mathrm{DC}(\mathrm{mm})$ & $\mathrm{H}(\mathrm{cm})$ & $\mathrm{H} / \mathrm{DC}$ & $\mathrm{DC}(\mathrm{mm})$ & $\mathrm{H}(\mathrm{cm})$ & $\mathrm{H} / \mathrm{DC}$ & $\mathrm{DC}(\mathrm{mm})$ & $\mathrm{H}(\mathrm{cm})$ & $\mathrm{H} / \mathrm{DC}$ \\
\hline T1 & $2,83 \mathrm{ab}$ & $17,08 \mathrm{c}$ & $6,11 \mathrm{c}$ & $3,55 \mathrm{ab}$ & $23,64 \mathrm{ab}$ & $6,72 \mathrm{a}$ & $5,16 \mathrm{a}$ & $26,32 \mathrm{~cd}$ & $5,11 \mathrm{~b}$ \\
\hline $\mathrm{T} 2$ & $2,62 \mathrm{bc}$ & $19,01 \mathrm{abc}$ & $7,36 \mathrm{a}$ & $3,57 \mathrm{ab}$ & $24,75 \mathrm{ab}$ & $7,15 \mathrm{a}$ & $4,95 \mathrm{ab}$ & $29,61 \mathrm{ab}$ & $6,02 \mathrm{a}$ \\
\hline $\mathrm{T} 3$ & $2,84 \mathrm{ab}$ & $19,34 \mathrm{ab}$ & $6,91 \mathrm{abc}$ & $3,91 \mathrm{a}$ & $24,93 \mathrm{ab}$ & $6,43 \mathrm{a}$ & $5,24 \mathrm{a}$ & $29,78 \mathrm{a}$ & $5,73 \mathrm{ab}$ \\
\hline $\mathrm{T} 4$ & $2,93 \mathrm{a}$ & $18,11 \mathrm{abc}$ & $6,21 b c$ & $3,83 \mathrm{ab}$ & $23,01 \mathrm{ab}$ & $6,09 \mathrm{a}$ & $5,18 \mathrm{a}$ & $27,53 \mathrm{bc}$ & $5,37 \mathrm{ab}$ \\
\hline $\mathrm{T} 5$ & $2,49 \mathrm{c}$ & $17,25 \mathrm{bc}$ & $7,17 \mathrm{ab}$ & $3,35 \mathrm{~b}$ & $22,47 \mathrm{~b}$ & $6,80 \mathrm{a}$ & $4,53 \mathrm{~b}$ & $24,29 \mathrm{~d}$ & $5,40 \mathrm{ab}$ \\
\hline $\mathrm{T} 6$ & $2,82 \mathrm{ab}$ & $20,11 \mathrm{a}$ & $7,27 \mathrm{a}$ & $3,92 \mathrm{a}$ & $25,63 \mathrm{a}$ & $6,62 \mathrm{a}$ & $5,33 \mathrm{a}$ & $30,16 \mathrm{a}$ & $5,72 \mathrm{ab}$ \\
\hline $\mathrm{T} 7$ & $2,91 \mathrm{ab}$ & $20,12 \mathrm{a}$ & 7,01 abc & $4,07 \mathrm{a}$ & $25,31 \mathrm{ab}$ & $6,24 \mathrm{a}$ & $5,28 \mathrm{a}$ & $30,10 \mathrm{a}$ & $5,74 \mathrm{ab}$ \\
\hline CV (\%) & 4,8 & 4,79 & 6,62 & 5,94 & 4,72 & 8,59 & 4,55 & 3,21 & 4,79 \\
\hline Média & 2,78 & 18,72 & 6,86 & 3,74 & 24,25 & 6,58 & 5,09 & 28,25 & 5,58 \\
\hline
\end{tabular}

Médias seguidas de mesma letra nas colunas não diferem estatisticamente a $5 \%$ de probabilidade $(\mathrm{p}<0,05)$ pelo teste de Tukey, em que CV (\%) é o coeficiente de variação. T1- substrato comercial Bioplant ${ }^{\circledR}(100)$; T2- esterco bovino mais casca de arroz carbonizada (50:50); T3- esterco bovino mais areia (50:50); T4- esterco bovino mais areia (25:75); T5- substrato comercial mais casca de arroz carbonizada (25:75); T6- substrato comercial mais esterco bovino (25:75); e T7- areia mais esterco bovino $(25: 75)$.

Means followed by the same letter in the columns do not differ by Tukey test statistically at $5 \%$ probability $(\mathrm{p}<0,05)$ where $\mathrm{CV}$ $(\%)=$ coefficient of variation, referred to treatment: $\mathrm{T} 1-$ Commercial substrate Bioplant $^{\circledR}, \mathrm{T} 2-$ Manure bovine + Carbonized rice husk (50:50), T3 - Manure bovine + sand (50:50), T4 - Manure bovine + sand (25:75), T5 - Commercial substrate + Carbonized rice husk (25:75), T6 - Commercial substrate + Manure bovine (25:75), T7 - Sand + Manure bovine (25:75).

Aos 90 dias, não houve diferenças estatísticas entre os tratamentos, exceto o T5, composto de substrato comercial mais casca de arroz carbonizada (25:75), com 3,35 mm de diâmetro do coleto. O T7, areia mais esterco bovino (25:75), com 4,07 mm de diâmetro do coleto, foi o que apresentou melhores resultados. Dados semelhantes foram encontrados por Martins Filho et al. (2015), em que, avaliando o desenvolvimento da pupunheira espécie Bactris gasipaes Kunth., verificaram que substratos com predominância de esterco bovino obtiveram melhores resultados no desenvolvimento do diâmetro do coleto.

Já aos 120 dias após o plantio, o T1, substrato comercial Bioplant ${ }^{\circledR}(100)$, o T3, esterco bovino mais areia (50:50), o T4, esterco bovino mais areia (25:75), o T6, substrato comercial mais esterco bovino (25:75) e o T7, areia mais esterco bovino (25:75), foram os que tiveram melhores desenvolvimentos do diâmetro do coleto, com $5,16,5,24,5,18,5,33$ e $5,28 \mathrm{~mm}$, respectivamente. Novamente, o T5, composto por substrato comercial mais casca de arroz carbonizada (25:75), foi considerado o pior tratamento aos 120 dias, com 4,53 mm de diâmetro do coleto (Tabela 1).
Segundo Silva et al. (2015), substratos que possuem em sua composição altas quantidades de compostos orgânicos influenciam positivamente no desenvolvimento no coleto de mudas. Em seu estudo, os autores avaliaram a influência do lodo de esgoto no desenvolvimento de Euterpe edulis Mart., em que os resultados comprovaram a influência positiva desse composto no desenvolvimento de palmeiras.

Gonzaga et al. (2016), estudando recipientes e substratos para produção de mudas de Hymenaea courbaril L., observaram que os substratos contendo areia e solo proporcionaram as menores médias de desenvolvimento do diâmetro de colo, contradizendo os resultados encontrados na execução deste experimento.

Os tratamentos que obtiveram melhores resultados no crescimento em altura $(\mathrm{H}) \mathrm{em}$ todos os períodos avaliados foram T3, T6 e T7. Aos 60, 90 e 120 dias, o T3 obteve 19,34, 24,93 e 29,78 cm, o T6, 20,11, 25,63 e 30,16 cm, e o T7, 20,12, 25,31 e 30,10 cm de altura, respectivamente. O menor valor de altura aos 60 dias foi encontrado no T1, com 17,08 cm, e aos 90 e 120 dias no T5, com 22,47 e 24,29 cm, respectivamente (Tabela 1 ). 
SOUSA, R.M. et al. Produção de mudas de açaí..

A relação altura/diâmetro (H/DC) aos 60 dias obteve resultados superiores no T2 e no T6. Os demais tratamentos não diferiram estatisticamente entre si. Esses dados foram observados aos 60, 90 e 120 dias, exceto no $\mathrm{T} 1$, composto de $100 \%$ de substrato comercial Bioplant $^{\circledR}$, que, aos 120 dias, obteve 5,11 entre a relação altura/diâmetro (H/DC) (Tabela 1). Cabe ressaltar que a relação H/DC, denominada quociente de robustez, fornece informações de quão delgada está a muda, sendo considerado um índice preciso, pois exprime o equilíbrio do crescimento (Silva et al., 2015).

Segundo Figuerêdo et al. (2014), proporções inferiores a $51 \%$ de casca de arroz carbonizada na composição de substratos proporcionaram melhores resultados no desenvolvimento da muda de Eucalyptus globulus Labill, corroborando os resultados obtidos no presente estudo na relação $\mathrm{H} / \mathrm{DC}$, em que o T2 possui em sua composição $50 \%$ de casca de arroz carbonizada.
Santos et al. (2014) estudaram o crescimento de mudas de Parapiptadenia rigida (Benth.) Brenan sob diferentes substratos e constataram que a composição $60 \%$ lodo de esgoto mais $40 \%$ casca de arroz carbonizada destacou-se no desenvolvimento das mudas, proporcionando maiores médias das características de crescimento.

Os resultados de MSPA, MSR, MST e RMSPAR não diferiram entre si, ou seja, os sete tratamentos testados não obtiveram diferenças significativas na análise estatística (Tabela 2). Nakazono et al. (2001) afirmam que indivíduos de Euterpe oleracea Mart., quando submetidos a um crescimento sob quantidade de luz de 20, 30, 50 e 70\%, não diferem em área foliar e distribuição de biomassa entre raiz e parte aérea, corroborando os resultados obtidos no presente estudo, no qual o experimento foi conduzido sob sombrite de $50 \%$ de luminosidade.

Tabela 2. Valores obtidos aos 120 dias de semeadura de Euterpe oleracea Mart. produzida em diferentes substratos, em que MSPA é a massa seca da parte aérea, MSR é a massa seca da raiz, MST é a massa seca total, RMSPAR é a massa seca da parte aérea e a massa seca da raiz e IQD é o índice de qualidade de Dickson.

Table 2. Values obtained at 120 days of sowing of Euterpe oleracea Mart. produced on substrates different, where MSPA-dry mass of the aerial part, MSR- dry mass of roots, MST- total dry mass, RMSPAR- relation between the aerial part dry mass and root dry mass dry mass of roots and IQD- Dickson quality index.

\begin{tabular}{clllll}
\hline Tratamento & MSPA & MSR & MST & RMSPAR & IQD \\
\hline T1 & $4,77 \mathrm{a}$ & $4,02 \mathrm{a}$ & $8,78 \mathrm{a}$ & $1,24 \mathrm{a}$ & $1,39 \mathrm{a}$ \\
T2 & $4,45 \mathrm{a}$ & $3,97 \mathrm{a}$ & $8,42 \mathrm{a}$ & $1,20 \mathrm{a}$ & $1,18 \mathrm{~b}$ \\
T3 & $4,49 \mathrm{a}$ & $4,23 \mathrm{a}$ & $8,72 \mathrm{a}$ & $1,09 \mathrm{a}$ & $1,29 \mathrm{ab}$ \\
T4 & $4,70 \mathrm{a}$ & $4,08 \mathrm{a}$ & $8,78 \mathrm{a}$ & $1,20 \mathrm{a}$ & $1,35 \mathrm{ab}$ \\
T5 & $4,42 \mathrm{a}$ & $4,51 \mathrm{a}$ & $8,93 \mathrm{a}$ & $0,98 \mathrm{a}$ & $1,41 \mathrm{a}$ \\
T6 & $4,55 \mathrm{a}$ & $4,50 \mathrm{a}$ & $9,05 \mathrm{a}$ & $1,02 \mathrm{a}$ & $1,36 \mathrm{ab}$ \\
T7 & $4,59 \mathrm{a}$ & $4,47 \mathrm{a}$ & $9,06 \mathrm{a}$ & $1,03 \mathrm{a}$ & $1,35 \mathrm{ab}$ \\
CV $(\%)$ & 5,95 & 7,4 & 3,93 & 14,94 & 6,13 \\
Média & 4,57 & 4,25 & 8,82 & 1,11 & 1,33 \\
\hline
\end{tabular}

Médias seguidas de mesma letra nas colunas não diferem estatisticamente a $5 \%$ de probabilidade $(\mathrm{p}<0,05)$ pelo teste de Tukey, em que CV (\%) é o coeficiente de variação. T1- substrato comercial Bioplant ${ }^{\circledR}$ (100); T2- esterco bovino mais casca de arroz carbonizada (50:50); T3- esterco bovino mais areia (50:50); T4- esterco bovino mais areia (25:75); T5- substrato comercial mais casca de arroz carbonizada (25:75); T6- substrato comercial mais esterco bovino (25:75); e T7- areia mais esterco bovino (25:75).

Means followed by the same letter in the columns do not differ by Tukey test statistically at $5 \%$ probability $(\mathrm{p}<0,05)$ where $\mathrm{CV}(\%)=$ coefficient of variation, referred to treatment: $\mathrm{T} 1-$ Commercial substrate Bioplant ${ }^{\circledR}, \mathrm{T} 2-$ Manure bovine + Carbonized rice husk (50:50), T3 - Manure bovine + sand (50:50), T4 - Manure bovine + sand (25:75), T5 - Commercial substrate + Carbonized rice husk (25:75), T6 - Commercial substrate + Manure bovine (25:75), T7 - Sand + Manure bovine (25:75). 
Em relação à característica índice de qualidade de Dickson (IQD), foram encontrados resultados que variaram de 1,18 a 1,41. Caldeira et al. (2012) afirmam que, quanto maior o valor desse parâmetro, melhor é a qualidade da muda, ou seja, os melhores valores foram obtidos por T1 $(1,39 \mathrm{~cm})$ e T5 $(1,41 \mathrm{~cm})$. Pinto et al. (2016) calcularam a qualidade das mudas levando em consideração a robustez e a biomassa delas, obtendo, assim, o equilíbrio da muda.

O T7, composto por areia mais esterco bovino (25:75), e o T4, esterco bovino mais areia (25:75), obtiveram resultados idênticos para o IQD, com 1,35 cm; porém, cabe ressaltar que T7 e T4 foram compostos por areia mais esterco bovino, diferindo apenas na porcentagem de concentração de cada substrato utilizado. Dessa forma, pode-se inferir que $\mathrm{T} 7$, por ter maior porcentagem de esterco bovino (75\%), notadamente possui maior microporosidade e maior retenção de água. Segundo Melém Junior e Queiroz (2011), os agricultores familiares e ribeirinhos do bioma Amazônico, fisionomia várzea, plantam açaí nessas áreas onde os solos são porosos, ricos em nutrientes e temporariamente bem úmidos. Já o T4 possui características opostas, isto é, maior macroporosidade e menor retenção de água, causado pela presença de $75 \%$ de areia, ou seja, representando solos da fisionomia terra firme presente no bioma Amazônico.

As variáveis MSPA, MSR e MST apresentaram maiores valores no T1, T4 e T7, sendo os dois últimos compostos por esterco bovino (Tabela 2). Carvalho Filho et al. (2003) recomendaram uma mistura contendo solo, areia e esterco bovino (1:2:1) para produção de mudas de jatobá (Hymenaea courbaril L.) aos 180 dias de idade. Resultados semelhantes foram obtidos no presente estudo, evidenciando o fato de que substratos contendo matéria orgânica são os mais indicados para produção de mudas de Euterpe oleracea Mart.

A relação MSPAR é um índice eficiente para elucidar o padrão de qualidade das mudas, sendo recomendado valores próximos a 2 (Carneiro, 1995). Neste estudo, com exceção do T5, substrato comercial mais casca de arroz carbonizada (25:75), que apresentou 0,98 de relação MSPAR, todos os demais tratamentos obtiveram valores maiores que 1, aproximando-se do ideal proposto pela literatura.

\section{CONCLUSÕES}

Os tratamentos com esterco bovino em sua composição, principalmente nas proporções de $50 \%$ de esterco bovino mais $50 \%$ de areia (T3), $25 \%$ de areia mais $75 \%$ de esterco bovino (T7) e $25 \%$ de substrato comercial Bioplant ${ }^{\circledR}$ mais $75 \%$ de esterco bovino (T6), proporcionaram os melhores crescimentos em altura e diâmetro do coleto e aumentaram a produção de massa seca total em mudas de Euterpe oleracea Mart.

\section{REFERÊNCIAS BIBLIOGRÁFICAS}

CALDEIRA, M.V.W. et al. Biossólido na composição de substrato para a produção de mudas de Tectona grandis. Floresta, v. 42, n. 1, p. 77-84, 2012. http:// dx.doi.org/10.5380/rf.v42i1.26302.

CARnEIRO, J.G.A. Produção e controle de qualidade de mudas florestais. Curitiba: Editora UFPR/FUPEF, 1995, $451 \mathrm{p}$.

CARVALHO FILHO, J.L.S. et al. Produção de mudas de jatobá (Hymenaea courbaril L.) em diferentes ambientes, recipientes e composições de substratos. Cerne, v. 9, n. 1, p. 109-118, 2003.

CARVALHO, L.C.N. Produção de mudas de açaí sob diferentes níveis de depleção de água associada a doses de um polímero hidroabsorvente. $2016.79 \mathrm{f}$. Dissertação (Mestrado em Ciências) - Escola Superior de Agricultura "Luiz de Queiroz", Universidade de São Paulo, Piracicaba, 2016.

CONFORTO, E.C.; CONTIN, D.R. Desenvolvimento do açaizeiro de terra firme, cultivar Pará, sob atenuação da radiação solar em fase de viveiro. Bragantia, v. 68, n. 4, p. 979-983, 2009. http://dx.doi.org/10.1590/ S0006-87052009000400018.

FERREIRA, D.F. Sisvar: a computer statistical analysis system. Ciência e Agrotecnologia, v. 35, n. 6, p. 1039-1042, 2011. http://dx.doi.org/10.1590/ S1413-70542011000600001.

FIGUERÊDO, K.S. et al. Adição de casca de arroz carbonizada em diferentes substratos para produção de mudas de Eucalyptus globulus. Journal of Biotechnology and Biodiversity, v. 5, n. 1, p. 7178, 2014. 
SOUSA, R.M. et al. Produção de mudas de açaí..

GONZAGA, L.M. et al. Recipientes e substratos para a produção de mudas de jatobá (Hymenaea courbaril L.). Revista Brasileira de Agropecuária Sustentável, v. 6, n. 1, p. 64-73, 2016. http://dx.doi. org/10.21206/rbas.v6i1.309.

MARTINS FILHO, S. et al. Diferentes substratos afetando o desenvolvimento de mudas de palmeiras/ different substrata affecting the development of palm tree seedlings. Ceres, v. 54, n. 311, p. 80-86, 2015.

MEDEIROS, M.C.L.; MEDEIROS D.C.; LIBERALINO FILHO, J. Adubação foliar na cultura da rúcula em diferentes substratos. Revista Verde, v. 2, n. 2, p. 158-161, 2007.

MELÉM JUNIOR, N.J.; QUEIROZ, J.A.L. Plantio de açaizeiro em terra firme. Macapá: Embrapa Amapá, 2011. 26 p.

MENDONÇA, V.C.M.; BERNARDES, R.H.; DEL BIANCHI, V.L. Impacto do surto da doença de chagas na comercialização do açaí (Euterpe oleracea Mart.) no município de Pinheiro - MA. Revista Sodebras, v. 9, n. 100, p. 174-178, 2014.

NAKAZONO, E.M. et al. Early growth of Euterpe edulis Mart. in different light enviroments. Revista Brasileira de Botanica. Brazilian Journal of Botany, v. 24, n. 2, p. 173-179, 2001.

NEVES, L.T.B.C. et al. Quality of fruits manually processed of açaí (Euterpe oleracea Mart.) and bacaba (Oenocarpus bacaba Mart.). Revista Brasileira de Fruticultura, v. 37, n. 3, p. 729-738, 2015.

OLIVEIRA, J.F. et al. Análises físico-química e microbiológica de palmito em conserva do tipo Açaí (Euterpe oleracea). Revista Brasileira de Higiene e Sanidade Animal, v. 11, n. 1, p. 8-18, 2017.

PINTO, A.V.F. et al. Efeitos de substratos e recipientes na qualidade das mudas de Tabebuia aurea (Silva Manso) Benth. \& Hook. F. Ex S. Moore. Revista Biociências, v. 22, n. 1, p. 100-109, 2016.

PORTINHO, J.A.; ZIMMERMANN, L.M.; BRUCK, M.R. Efeitos benéficos do açaí. Journal of Nutrology, v. 5, n. 1, p. 15-20, 2012.
RODRIGUES, R.A.; AMARAL, E.A.; GALVÃO, A.S. Acarofauna em açaizeiro (Euterpe oleracea Mart.) conduzido em diferentes sistemas de cultivo. RevistaAgro@mbiente. Online (Bergheim), v. 10, n. 3, p. 273-281, 2016.

SANTOS, F.E.V.; CALDEIRA, M.V.W.; KUNZ, S.H. Qualidade de mudas de Parapiptadenia rigida (Benth.) Brenan produzidas em diferentes substratos com lodo de esgoto e casca de arroz. Revista Ecologia e Nutrição Florestal-ENFLO, v. 1, n. 2, p. 55-62, 2014. http://dx.doi.org/10.13086/2316-980x.v01n02a01.

SECRETARIA DO PLANEJAMENTO E DA MODERNIZAÇÃODA GESTÃO PÚBLICA-SEPLAN. Atlas do Tocantins: subsídios ao planejamento da gestão territorial. 2012. Disponível em: $<$ http:// web.seplan.to.gov.br/Arquivos/download/ZEE/ TO_AtlasTocantins2012/Atlas_do_Tocantins_2012. pdf>. Acesso em: 20 Mai. 2016.

SILVA, F.A.M. et al. Produção de mudas de juçara com resíduos agroindustriais e lodo de esgoto compostados/ juçara seedling production with agroindustry wastes and composted sewage sludge. Revista Brasileira de Engenharia de Biossistemas, v. 9, n. 2, p. 109121, 2015.

SOUSA, F.H.M. Regionalização climática de Thorntwhaite e Mather para o Estado do Tocantins. 2016. 113 f. Dissertação (Mestrado em Ciências Florestais e Ambientais) - Universidade Federal do Tocantins, Gurupi.

SOUZA JÚNIOR, J.O; CARMELLO, Q.A.C.; FARIA, J.C. Características químicas do lixiviado na fase de enraizamento de estacas de cacau em Substratos adubados com fósforo. Revista Brasileira de Ciência do Solo, v. 32, n. 4, p. 1573-1581, 2008. http://dx.doi.org/10.1590/S0100-06832008000400021.

ZAMBRANA, N.Y.P. et al. Diversity of palm uses in the westem Amazon. Biodiversity and Conservation, v. 16, n. 10 , p. $2771-2787,2007$. http://dx.doi. org/10.1007/s10531-007-9218-y. 